\title{
Where Can We Find Bypass Tract in Ebstein's Anomaly?; a Case of Successful Ablation of Bypass Tract in Ebstein's Anomaly
}

June Namgung, MD; Jae-Jin Kwak, $\mathrm{MD}$

Division of Cardiology, Department of Internal Medicine, Inje University Ilsan Paik Hospital, Goyang-si, Republic of Korea

Received: January 20, 2017

Revision Received: March 7, 2017

Accepted: March 22, 2017

Correspondence: June Namgung, MD, PhD

Division of Cardiology, Department of Internal

Medicine, Vison 21 Cardiac and Vascular Center,

Inje University Ilsan Paik Hospital, 170, Juhwa-ro,

Ilsanseo-gu, Goyang-si, Gyeonggi-do 10380

Republic of Korea

Tel: +82-31-910-9645 Fax: +82-31-910-7829

E-mail: jnamgung@paik.ac.kr

Copyright () 2017 The Official Journal of Korean Heart Rhythm Society Editorial Board \& MMK Co., Ltd.

\begin{abstract}
In patients with Ebstein's anomaly, the localization of accessory pathways may be impeded by abnormal local electrograms recorded along the atrialized right ventricle and by the presence of multiple accessory pathways. We report a case of 50-year-old man diagnosed with Ebstein's anomaly with Wolff-Parkinson-White syndrome who presented with recurrent palpitations. He was referred to our institution for radiofrequency catheter ablation of paroxysmal supraventricular tachycardia. Transthoracic echocardiography revealed the tricuspid valve displaced into the right ventricle, consistent with Ebstein's anomaly. The electrophysiology study showed a right posterolateral accessory pathway. The optimal ablation site was located not in the atrioventricular line of the atrialized ventricular portion, but in the original atrioventricular line.
\end{abstract}

Key Words: - Ebstein's anomaly -Wolff-Parkinson-White syndrome - accessory atrioventricular pathway

\section{Introduction}

Bidirectional pathways and associated Wolf-Parkinson-White (WPW) syndrome have been reported to be present in about 20-30\% of patients with Ebstein's anomalies. ${ }^{1}$ The vast majority of accessory pathways (APs) are located along the atrialized right ventricle $(\mathrm{ARV}){ }^{2}$ Radiofrequency ablation of the AP is widely accepted as first-line treatment for atrioventricular reciprocating tachycardia. However, the success rate in patients with Ebstein's anomaly is generally low and associated with an increased recurrence during follow-up. In these patients, precise AP localization may be impaired by the complex geometry of multiple APs and by their usual location along the malformed tricuspid valve annulus, a region where abnormal endocardial electrograms have been reported. In particular, the characteristics of the ARV make it difficult to localize the ablation site precisely. ${ }^{3}$

In this case, AP conduction was abolished not in the atrioventricular $(\mathrm{AV})$ junction of the $\mathrm{ARV}$, but in the original $\mathrm{AV}$ junction.

\section{Case Report}

A 50-year-old man with recurrent palpitations was referred to our hospital for treatment of WPW syndrome. The first 

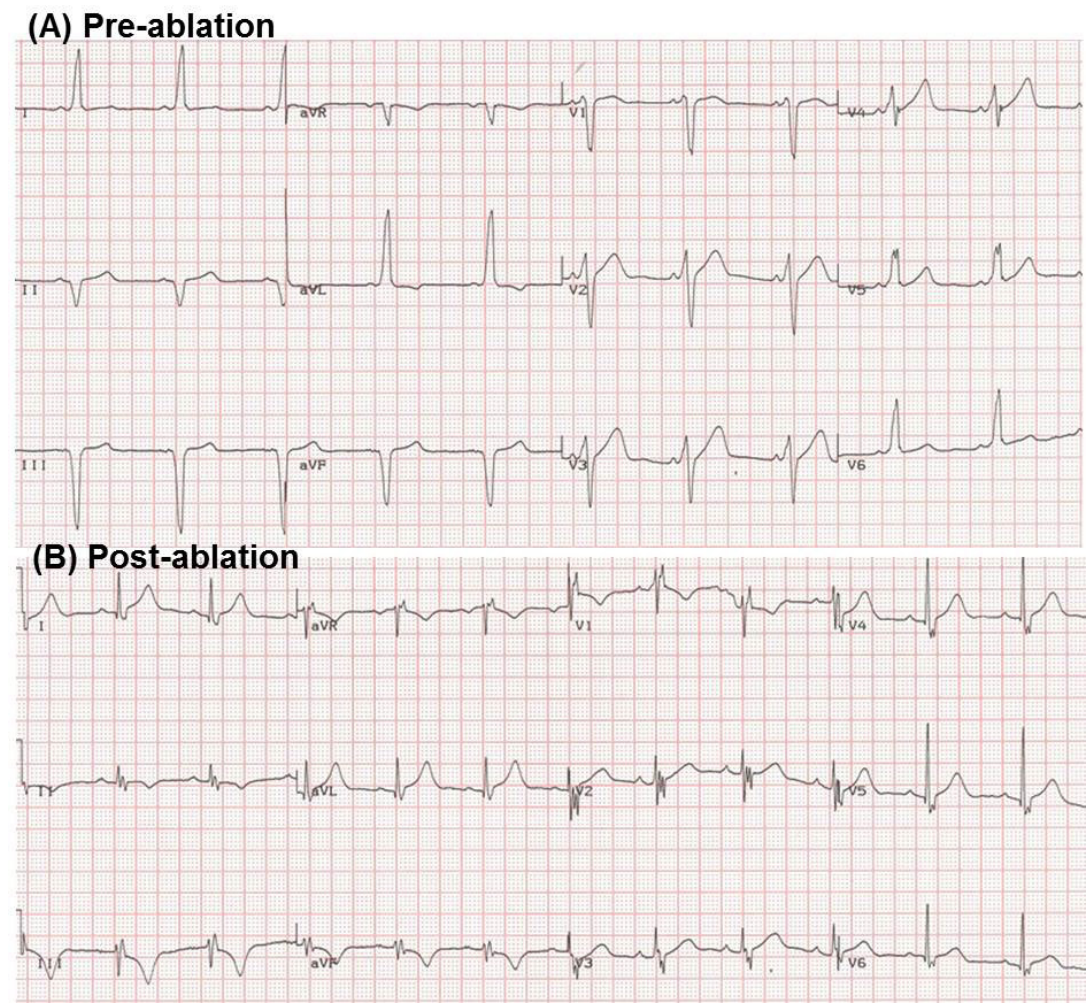

Figure 1. Twelve-lead electrocardiogram (A) pre-ablation: a short PR interval and delta waves are present in all leads; (B) post-ablation: after ablation of rapid conduction through an accessory pathway (AP), the delta wave disappeared.

palpitation attack occurred at the age of 40 years. On examination, he was noted to have a regular pulse, with a rate of $72 \mathrm{bpm}$, and normal jugular venous pressure. An ECG showed a normal sinus rhythm with ventricular pre-excitation suggestive of a right-sided AP. The PR interval was shortened at $0.10 \mathrm{sec}$, and the delta waves were most prominent in leads $\mathrm{I}$, aVL, and the lateral precordial leads (Figure 1). A chest radiograph showed a cardiothoracic ratio of $45 \%$ with a normal right atrium. Subsequent echocardiography revealed the apical displacement of the septal leaflet of the tricuspid valve from the insertion of the anterior leaflet of the mitral valve with minimal regurgitation (Figure 2). Marked enlargement of the right atrium and the ARV were present, features of Ebstein's anomaly. After written informed consent was obtained, an electrophysiological study and catheter ablation therapy were performed in off anti-arrhythmic medications. Under local anesthesia and using a sterile technique, one \#6 F quadripolar electrode catheter was percutaneously inserted into the right femoral vein and advanced using fluoroscopic guidance and placed in the His area. Using a similar technique, one \#6 F quadripolar electrode catheter (Saint Jude

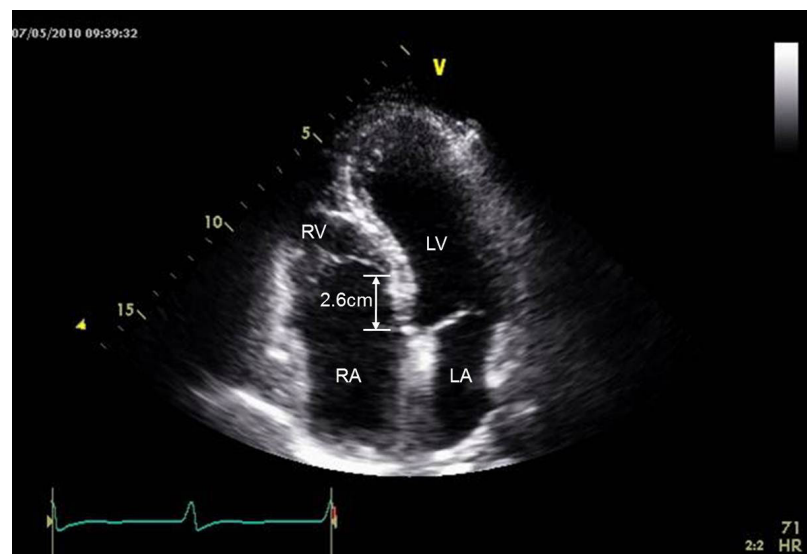

Figure 2. Apical four-chamber view of transthoracic echocardiography showing apical displacement of the septal tricuspid leaflet $2.6 \mathrm{~cm}$ apart from the original atrioventricular line. The increased separation between the anterior mitral and septal tricuspid leaflets is indicated by the arrowhead. $\mathrm{RA}$, right atrium; $\mathrm{RV}$, right ventricle 

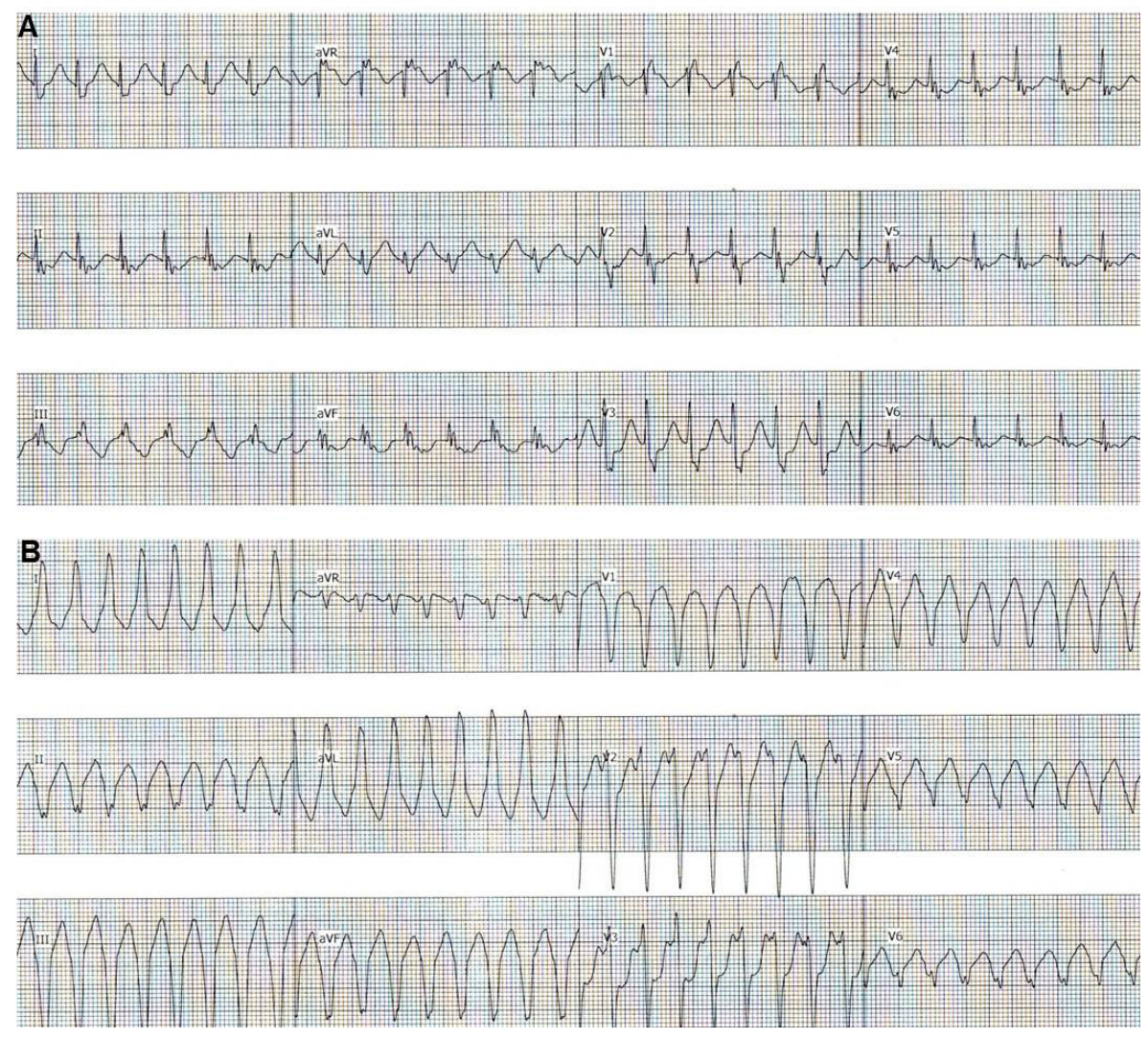

Figure 3. Narrow QRS (A) and wide QRS (B) atrioventricular reentrant tachycardia after programmed electrical stimulation from the high right atrium and the right ventricular apex

Medical, Minnetonka, Minnesota, USA) and one \#7 F duodecapolar deflectable Halo catheter (Saint Jude Medical, Minnetonka, Minnesota, USA) were percutaneously inserted into the left femoral vein and placed in the RV apex and the high RA to coronary sinus, respectively. Intracardiac electrograms were recorded. Conduction intervals were measured. Programmed stimulation was performed.

Tachycardias were easily induced, both by mechanically triggered premature depolarization during catheter positioning and during programmed stimulation from the right atrium. These tachycardias were both atrioventricular reentrant tachycardia (AVRT) with a narrow QRS complex (Figure 3A) and a wide QRS complex (Figure 3B). The rate of supraventricular tachycardia was $158 \mathrm{bpm}$ to $206 \mathrm{bpm}$. Recordings from the coronary sinus (CS) demonstrated that the earliest site of ventricular activation was between CS9, 10 and CS11, 12, indicating a right posterior AP (Figure 4A). A $7 \mathrm{~F}$ ablation catheter (Blazer ${ }^{\circ}$, Boston Scientific, Natick, Massachusetts, USA) was also inserted into the right femoral vein, and positioned at the site along the right posterior $\mathrm{AV}$ groove area where the earliest ventricular activity had been recorded. The atrial (A) and ventricular $(\mathrm{V})$ electrograms recorded from the ablation catheter tip were continuous, and the local ventricular activity preceded the onset of the delta wave on the surface ECG (Figure 4). Right anterior oblique views showed the tip of the ablation catheter in the original AV line, not in the AV line of the ARV portion (Figure 5A, 5B).

A radiofrequency current was delivered to the atrial insertion site of the right posterior AP via an ablation catheter. Within a few beats after the application of the radiofrequency energy, the delta wave on the ECG disappeared, and the PR interval normalized (Figure 4B). Prior to ablation, recordings from the CS 


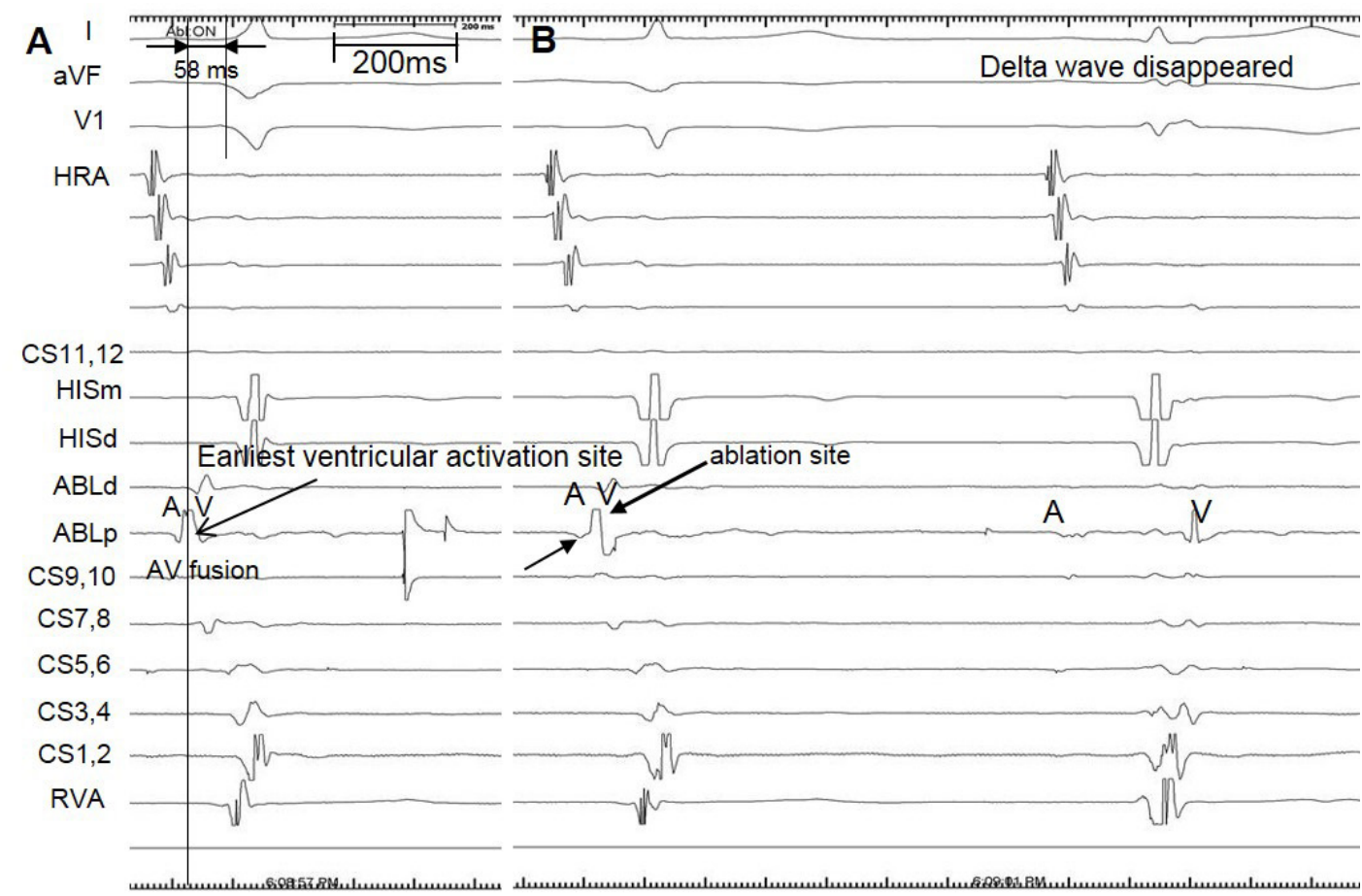

Figure 4. The ablation catheter was positioned along the right posterior atrioventricular groove area where it recorded fused atrial and ventricular electrograms during sinus rhythm (arrow) (A). The ventricular electrogram preceded the onset of the delta wave by 58 msec during sinus rhythm. Application of radiofrequency energy abolished the accessory pathway (B).

I, aVF, $V_{1}$ : surface electrocardiographic leads; HRA: intracardiac electrogram of the HRA; ABL: intracardiac electrogram of the ablation catheter; HIS: intracardiac electrogram of the bundle of His; CS: intracardiac electrogram of the coronary sinus; RVA: intracardiac electrogram of the right ventricular apex.
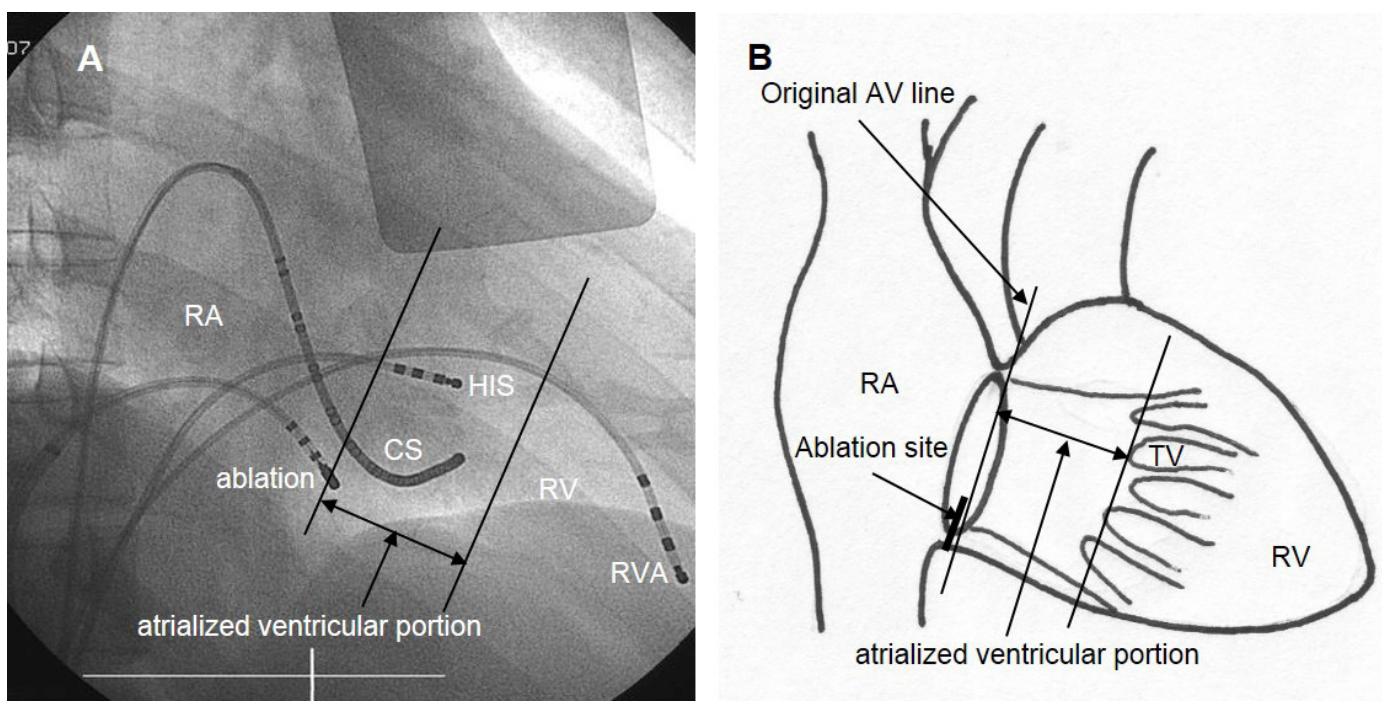

Figure 5. Fluoroscopic image of endocardial mapping catheters in the right anterior oblique view (RAO) (A) and schematic view (B). Multipolar catheters were positioned at the high right atrium (HRA), the bundle of His (HIS), the right ventricle (RV), and the coronary sinus (CS). The accessory pathway (AP) was located not in the atrioventricular line of the atrialized ventricular portion, but in the original atrioventricular line. Ablation of a manifest left posterior accessory pathway. 
catheter showed continuous A and V electrograms; after ablation there was a normal interval between the $\mathrm{A}$ and $\mathrm{V}$ electrograms. Neither pre-excitation nor tachycardia could be produced any more on 12-lead electrograms (Figure 1B).

The patient was discharged on the third day of hospital care with no prescription for any antiarrhythmics. The patient was asymptomatic at 16 months of follow-up, with no pre-excitation on surface ECG.

\section{Discussion}

This case describes a patient with Ebstein's anomaly who had a right posterior AP, located in the original AV groove. The clinically documented supraventricular tachycardia (SVT) were both orthodromic and antidromic AVRT via the right posterior AP.

Ebstein's anomaly is characterized by an abnormality of the tricuspid valve in which the septal leaflet and often the mural (posterior) leaflet attachment are displaced from the $\mathrm{AV}$ ring into the right ventricular cavity towards the apex. ${ }^{4}$ The septal leaflet of the tricuspid valve is situated apically, with discontinuity of the central fibrous body and the septal $\mathrm{AV}$ ring at its normal location. This condition may lead to a direct connection of the AV conduction system to the ventricular muscular structures, with the consequent formation of conditions favorable to the development of APs and pre-excitation. ${ }^{5}$ From 6\% to 36\% of patients with Ebstein's anomaly have more than one AP, and most APs are situated around the malformed tricuspid ring. ${ }^{6}$ Correct identification and treatment of APs are essential and may help to prevent sudden cardiac death. However, APs located along the ARV and abnormal morphology of endocardial activation potentials generated in this region make it difficult to identify a target site for radiofrequency (RF) current delivery. Such electrograms prevent the identification of AP activation potentials and of sites of earliest activation during antegrade or retrograde AP conduction. The characteristics of ARV might make it difficult to localize the ablation site precisely. Therefore, it is important to understand the characteristics of an abnormal electrogram in the ARV during catheter ablation therapy.
Not all cases of AP in Ebstein anomaly are applicable, in this case, the AP was localized in the area of the original AV line, not in the AV line of the ARV. After AP conduction was abolished, there was no evidence of delta waves and no inducible AVRT.

In conclusion, some patients with Ebstein's anomaly may experience life-threatening SVT episodes associated with accessory conduction pathways. Despite difficulties in identifying the localization of the AP associated with the abnormal electrograms that are commonly observed in the ARV, successful ablation is possible by more detailed electrical mapping against cardiac electrical conduction.

\section{References}

1) Krahn AD, Manfreda J, Tate RB, Mathewson FA, Cuddy TE. The natural history of electrocardiographic preexcitation in men. The Manitoba follow-up study. Ann Intern Med. 1992;116:456-460.

2) Cappato R, Schlüter M, Weiss C, Antz M, Koschyk DH, Hofmann T, Kuck KH. Radiofrequency Current Catheter Ablation of Accessory Atrioventricular Pathways in Ebstein's Anomaly. Circulation. 1996;94:376-383.

3) Triedman JK, Alexander ME, Love BA, Collins KK, Berul CI, Bevilacqua LM, Walsh EP. Influence of patient factors and ablative technologies on outcomes of radiofrequency ablation of intra-atrial re-entrant tachycardia in patients with congenital heart disease. $J$ Am Coll Cardiol. 2002;39:1827-1835.

4) Hebe J, Hansen P, Ouyang F, Volkmer M, Kuck KH. Radiofrequency catheter ablation of tachycardia in patients with congenital heart disease. Pediatr Cardiol. 2000;21:557-575.

5) de Groot NM, Schalij MJ, Zeppenfeld K, Blom NA, Van der Velde ET, Van der Wall EE. Voltage and activation mapping: how the recording technique affects the outcome of catheter ablation procedures in patients with congenital heart disease. Circulation. 2003;108:2099-2106.

6) Lee PC, Hwang B, Chen YJ, Tai CT, Chen SA, Chiang CE. Electrophysiologic characteristics and radiofrequency catheter ablation in children with Wolff-Parkinson-White syndrome. Pacing Clin Electrophysiol. 2006;29:490-495. 